\title{
NÍVEL DE ATIVIDADE FÍSICA E USO DE DROGAS LÍCITAS EM UNIVERSITÁRIOS
}

\section{Thomaz Talarico Neto ${ }^{1 *}$ Higino Carlos Hahns Júnior', Lucas Argenton Fernandes ${ }^{2}$, Claudia Teixeira-Arroyo ${ }^{2} \&$ Tiago Pedicini Ferreira da Silva ${ }^{2}$}

TALARICO NETO, T.; HAHNS JÚNIOR, H.C.; FENANDES, L.A.; TEIXEIRA-ARROYO, C.; \& SILVA, T.P.F. Nível de atividade física e uso de drogas lícitas em universitários. Perspectivas Online: Biológicas \& Saúde, v.10, n.34, p.58-67, 2020.

\section{RESUMO}

A entrada no Ensino Superior (ES) pode acarretar algumas mudanças na vida dos jovens, a integração com um novo grupo social somado a novas responsabilidades podem gerar uma diminuição em seus níveis de Atividade Física (AF). Ainda, fatores estressores podem motivá-los ao uso de drogas lícitas, causando prejuízos nesta população. Sendo assim, o objetivo do estudo foi avaliar o nível de AF, e o grau de dependência alcoólica e do tabaco em estudantes de uma rede de ensino superior. Para tal, participaram do estudo 204 universitários $(49,5 \%$ homens e 50,5\% mulheres) com média de idade de $22 \pm 5$ anos. O nível de AF foi avaliado através do International Physical Activity Questionnaire (IPAQ) versão longa. Para Alcoolismo e tabagismo, foram utilizadas as ferramentas CAGE e FAGERSTROM, respectivamente. A aplicação dos instrumentos foi feita via online, na plataforma Google docs. Como resultados, a maioria $(71 \%)$ dos estudantes trabalham de forma remunerada. Grande parte alcançou um nível "Alto" de atividade física $(64,21 \%)$. Relacionado ao consumo de álcool, 91,7\% apresentaram classificação "Não Abusiva", porém, $24,5 \%$ afirmaram ter aumentado o consumo após ingresso no ES. Sobre uso de Nicotina, 96,5\% dos participantes declararam não fumar, dentre os fumantes, $42,85 \%$ apresentaram "Alto Nível" de dependência, e ainda 32\% aumentaram o uso após o ingresso no ES. Pode-se concluir que os avaliados são em prevalência ativos, e a maioria não apresenta uso abusivo de álcool e tabaco. Ainda, o bom número de não fumantes pode ser fruto de políticas públicas antitabaco.

Palavras-chave: Atividade Física; Alcoolismo; Tabagismo; Universitários.

\footnotetext{
${ }^{1}$ Universidade de São Paulo, Escola de Edicação Física e Esporte de Ribeirão Preto - EEFERP/USP - Vila Monte Alegre, Ribeirão Preto - SP, CEP: 14040900, Brasil.

${ }^{2}$ Centro Universitário UNIFAFIBE, Rua Prof. Orlando França de Carvalho, 325, Bebedouro - SP, CEP: 14701-070, Brasil. $(*)$ e-mail: thomaz.talarico.edf@gmail.com
} 
Revista Perspectivas Online: Biológicas \& Saúde August/2020, v.10, n.34, p.58-67 ISSN: 2236-8868 (Online)

DOI: $10.25242 / 8868103420202042$

\title{
LEVEL OF PHYSICAL ACTIVITY AND THE USE OF LEGAL DRUGS BY UNIVERSITY STUDENTS
}

\section{Thomaz Talarico Neto ${ }^{1 *}$ Higino Carlos Hahns Júnior', Lucas Argenton Fernandes', Claudia Teixeira-Arroyo ${ }^{2}$ \& Tiago Pedicini Ferreira da Silva ${ }^{2}$}

TALARICO NETO, T.; HAHNS JÚNIOR, H.C.; FENANDES, L.A.; TEIXEIRA-ARROYO, C.; \& SILVA, T.P.F. Level of physical activity and the use of legal drugs by university students. Perspectivas Online: Biológicas \& Saúde, v.10, n.34, p.58-67, 2020.

\begin{abstract}
Entry into Higher Education (HE) can lead to some changes in the lives of young people, integration with a new social group added to new responsibilities can generate a decrease in their levels of Physical Activity (PA). Still, stressors can motivate them to use licit drugs, causing harm to the health of this population. Therefore, the objective of this study was to evaluate the level of PA, and the degree of alcohol and tobacco dependence in students of a higher education network. For this, took part of the study 204 college students $(49.5 \%$ men and $50.5 \%$ women) with a mean age of 22 \pm 5 years. The AF level was assessed using the International Physical Activity Questionnaire (IPAQ) long form. For alcoholism and smoking, the CAGE and FAGERSTROM tools were used, respectively. The application of the

instruments was done online, in the platform Google docs. As results, the majority $(71 \%)$ of students work on a paid basis. Much of it reached a "High" level of physical activity (64.21\%). Regarding alcohol consumption, $91.7 \%$ presented a "Non-Abusive" classification, however, $24.5 \%$ reported having increased their consumption after enrollment in ES. Regarding the use of nicotine, $96.5 \%$ of the participants stated that they did not smoke. Among smokers, $42.85 \%$ presented a "High Level" of dependence, and 32\% increased their use after entering the ES. It can be concluded that those evaluated are in prevalence active levels of PA, and most do not show abusive use of alcohol and tobacco. Moreover, the good number of nonsmokers may be the result of public anti-tobacco policies.
\end{abstract}

Keywords: Physical Activity; Alcoholism; Smoking; College Students.

\footnotetext{
${ }^{1}$ Universidade de São Paulo, Escola de Edicação Física e Esporte de Ribeirão Preto - EEFERP/USP - Vila Monte Alegre, Ribeirão Preto - SP, CEP: 14040900, Brasil.

${ }^{2}$ Centro Universitário UNIFAFIBE, Rua Prof. Orlando França de Carvalho, 325, Bebedouro - SP, CEP: 14701-070, Brasil. (*) e-mail: thomaz.talarico.edf@gmail.com
} 


\section{INTRODUÇÃO}

O ingresso no Ensino Superior (ES) é um período de extrema importância na vida dos jovens. O convívio com um novo grupo social, que muitas vezes vem acompanhado de uma maior liberdade, pode ser um divisor de águas, levando a escolhas determinantes para o futuro, sejam estas de cunho positivo ou negativo. Nesta acepção, a Organização Mundial da Saúde (OMS) inclui dois grandes campos como primordiais para uma vida saudável, sendo estes divididos em fatores modificáveis (atividade física, alimentação e consumo de drogas lícitas), e fatores não modificáveis (sexo, idade e herança genética) (OMS, 2005).

De fato, a vida universitária pode acarretar algumas mudanças comportamentais (fatores modificáveis), e nesta toante, pesquisas têm se preocupado com o nível de Atividade Física (AF) desta população, em vista que, baixos níveis da mesma estão relacionados com doenças, tais como diabetes, obesidade, infarto agudo do miocárdio, doença arterial coronariana e até mesmo câncer, podendo assim aumentar gastos no setor público de saúde, consequentes destas desordens (OMS, 2005; PITANGA; LESSA, 2005; TROLLELAGERROS et al., 2005).

A partir de uma revisão de literatura, pode-se observar um crescente aumento de publicações a partir de 2006 sobre o nível de AF em universitários, principalmente nas regiões Nordeste e Sudeste (SOUSA, 2011). Ainda, a mesma revisão apontou que estudantes apresentaram baixos níveis de AF em todos os domínios: lazer, deslocamento, atividades domésticas e ocupacionais (SOUSA, 2011). Em adição, outros estudos mostraram que os discentes que cursavam o período noturno, apresentaram um menor nível de AF quando comparados aos outros turnos, talvez pelo fato de conciliarem a dupla jornada de estudos e trabalho (FONTES; VIANNA, 2009).

Associados aos problemas de baixos níveis de AF, universitários ainda encaram questões de consumo de álcool e tabaco, ou ainda o policonsumo simultâneo (álcool + tabaco), como mostram os dados do I levantamento sobre o uso de álcool, tabaco e outras drogas entre universitários de 27 capitais brasileiras, onde a pesquisa apontou um maior consumo dessas substâncias em universitários do que na população geral (ANDRADE; DUARTE; OLIVEIRA, 2010). Ainda, existe todo um aparato no que tange as políticas públicas no Brasil, onde se tem criado estratégias para diminuição do consumo destas substâncias, e os resultados mostram-se positivos como no caso do tabaco, apontando uma progressiva e sustentada redução no número percentual de fumantes em todo o país entre 1990 a 2015, como publicado por um grande estudo (REITSMA et al., 2017).

Contudo, ainda é necessário um olhar mais apurado justamente nas populações mais tendenciosas ao uso, como caso de universitários, uma vez que, o tabaco tem relação direta com $30 \%$ das mortes por câncer, além de desenvolver doenças cardíacas, circulatórias e respiratórias, também podendo levar a desordens psíquicas como transtorno de déficit de atenção e hiperatividade (TDAH), atenuação do desempenho intelectual, depressão e ainda estimular o uso de álcool (ANDRADE; DUARTE; OLIVEIRA, 2010; DEHEINZELIN et al., 2005; MALBERGIER; OLIVEIRA JUNIOR, 2005; MILLAR; LOCKER, 2007; OLIVEIRA; VALENTE; LEITE, 2008). Ainda, estudos experimentais relatam que a fumaça do cigarro pode acarretar um processo inflamatório e espessamento dos alveólos pulmonares (CRISCI et al., 2015).

Em relação ao consumo de álcool, este tem sido relacionado com complicações mais frequentes de saúde, onde pacientes com problemas relacionados ao uso desta substância

Persp. online: biol. \& saúde, Campos dos Goytacazes, 34 (10) 58-67, 2020 https://ojs3.perspectivasonline.com.br 
utilizaram três vezes mais o serviço de saúde do que aqueles que não apresentavam problemas com a bebida (ODO et al., 2000). Ainda, um estudo realizado com usuários do Centro de Atenção Psicossocial Álcool e Drogas (CAPSad) demosntra que o álcool é a substância psicoativa de maior prevalência entre os sujeutos (CONSTANTINO; BATISTA, 2012).

Ainda, o álcool atinge zonas de perigo que expõem a população em geral. Direcionado aos jovens, estes quando alcoolizados, tendem a se envolver mais em relações sexuais sem proteção, desta forma, se expondo a doenças sexualmente transmissíveis e até mesmo a gravidez com maior frequência (HUIZINGA; LOEBER; THORNBERRY, 1993). Ainda, a embriaguez acarreta vários déficits acadêmicos, inclusive notas mais baixas (HUIZINGA; LOEBER; THORNBERRY, 1993). Em outro estudo, foi interessante o fato que, os estudantes que mais frequentavam a biblioteca eram os que possuíam menores índices de consumo de álcool, tabaco e outras drogas (SILVA et al., 2006).

Diante de todo o referencial teórico, baixos níveis de AF relacionados ao tabagismo e alcoolismo podem afetar profundamente a vida dos jovens universitários em um nível biopsicossocial, uma vez que, hábitos adquiridos neste período podem perdurar pelo resto de suas vidas. Sendo assim, o objetivo deste estudo foi avaliar o nível de AF juntamente com grau de dependência do álcool e tabaco em uma população universitária de uma rede privada de ensino superior no interior de São Paulo, a fim de compreender o perfil desta população.

\section{METODOLOGIA}

Este estudo é uma pesquisa de campo transversal de natureza descritiva. O Projeto foi aprovado pelo Comitê de Ética do Centro Universitário UNIFAFIBE (CAAE: 74875817.4.0000.5387), garantindo assim que todos os cuidados éticos estão em conformidade como a com a Resolução 466/12.

\subsection{Participantes}

Participaram do estudo 204 universitários de ambos os sexos, com uma média de idade de $22 \pm 5$ anos, de 14 diferentes cursos de graduação de um Centro Universitário no interior de São Paulo. Como critérios de inclusão, o aluno devia estar regularmente matriculado na instituição na qual foi desenvolvida a pesquisa e também possuir idade maior ou igual a 18 anos.

\subsection{Instrumentos de pesquisa}

Para a realização deste estudo foram utilizados três (3) ferramentas:

a) Questionário de nível de AF. Foi utilizado o International Physical Activity Questionnaire IPAQ versão longa. Esta ferramenta quantifica os dias e minutos das atividades realizadas pelos participantes na última semana, considerando atividades ocupacionais, de lazer, locomoção e trabalho doméstico. Este instrumento é de fácil aplicação e de grande utilidade na literatura, sendo também

Persp. online: biol. \& saúde, Campos dos Goytacazes, 34 (10) 58-67, 2020

https://ojs3.perspectivasonline.com.br 
validado para a população brasileira (MATSUDO et al., 2001). Por meio do IPAQ é possível classificar o nível de atividade física do indivíduo em: alto, moderado e baixo;

b) Para mensurar o grau de dependência alcoólica foi utilizado o CAGE. Um questionário eficiente para diagnosticar a Síndrome de Dependência do Álcool (SDA). Esta ferramenta foi proposta por Ewing e Rouse (1970), traduzida e validada para o português (MASUR; MONTEIRO, 1983). Tem uma sensibilidade de $88 \%$ nos acertos de identificação para a porcentagem de alcoolistas. O questionário é composto por quatro questões que admitem como respostas "Sim" e "Não", sendo que, para duas respostas positivas, considera-se um quadro positivo para a SDA. Consiste em um método de rápida e fácil aplicação e pouco intimidativo, uma vez que, tais assuntos podem ser pessoais, e se tratando de respostas fechadas torna-o mais acessível;

c) Para dependência a nicotina foi utilizado o teste de FAGERSTROM. Este é um método utilizado mundialmente como ferramenta de avaliação para o grau de dependência a nicotina, substituindo testes mais caros, e sendo mais rápido e menos invasivo para com o indivíduo. É composto por seis perguntas, referentes ao uso do cigarro no que tange a quantidades, locais e circunstâncias. Foi desenvolvido, traduzido e validado para população brasileira (CARMO; PUEYO, 2002). O teste possui validade bem comprovada, uma vez que, os resultados demonstraram associações entre a ferramenta e as medidas bioquímicas relacionadas à quantidade de cigarros usadas através das dosagens de cotinina plasmática e urinária, e também quantidade de gás carbônico expirado.

\subsection{Procedimentos}

O processo de coleta de dados ocorreu durante dois meses, sendo que, as ferramentas utilizadas para mensurar nível de $\mathrm{AF}$, grau de dependência alcoólica e dependência à nicotina foram adequadas para um veículo online, na plataforma Google Docs. Uma vez que os cursos da instituição pesquisada utilizavam as salas de computação em suas aulas, os pesquisadores solicitaram a autorização dos coordenadores dos cursos para que quando a aula acontecesse nesta sala, pouco antes do início da mesma, os alunos pudessem responder os questionários nos computadores da instituição, onde o link já estava prontamente a disposição em todos os computadores. A primeira sessão deste questionário se dava pelo Termo de Consentimento Livre e Esclarecido (TCLE), onde, caso os estudantes aceitassem participar, era necessário clicar em uma caixa com o recado "concordo com os termos propostos" e só assim era possível iniciar a segunda sessão, onde lá teriam contato com as ferramentas de avaliação. Ainda, três pesquisadores permaneciam na sala para sanar quaisquer dúvidas durante a aplicação dos questionários.

\subsection{Análise dos dados}

Os dados foram analisados por meio de médias, desvios padrão e porcentagens. Quando realizado comparações entre homens e mulheres, foi utilizado o teste $t$ de Student. O programa estatístico utilizado para as análises foi o SPSS for Windows 18.0 e o nível de

Persp. online: biol. \& saúde, Campos dos Goytacazes, 34 (10) 58-67, 2020

https://ojs3.perspectivasonline.com.br 
significância adotado na análise foi de $\mathrm{p} \leq 0,05$.

\section{RESULTADOS}

Participaram do estudo 204 universitários de ambos os sexos, sendo 49,5\% homens e $50,5 \%$ mulheres, com uma média de idade de $22 \pm 5$ anos, de 14 diferentes cursos de graduação de um Centro Universitário no interior de São Paulo.

Os participantes desta pesquisa, em sua maioria $(71,1 \%)$, trabalham de forma remunerada, além de estudarem no período noturno, apresentando uma média diária de 4,9 $\pm 3,5$ horas de trabalho. Ainda, homens e mulheres mostraram semelhanças no perfil laboral. Quando analisados separadamente, 74\% dos homens possuem trabalho remunerado, e as mulheres $76,3 \%$, sendo números bem próximos. Ademais, as horas de trabalho são praticamente as mesmas entre os sexos, não obtendo diferença significativa.

Em relação ao Nível de AF, os universitários denotaram boas condições. A maioria apresentou um "Alto" nível de AF 64,21\%, seguido por "Moderado" 24,01\% e "Baixo" $11,78 \%$. Homens e Mulheres apresentaram classificações muito semelhantes em relação ao nível de AF quando analisados separadamente, posto que, a classificação dos homens segue: "Alto" 69\%, "Moderado" 20\% e "Baixo" 11\%. Já as mulheres apresentaram: "Alto" 59,61, "Moderado" 27,88 e "Baixo" 12,51. É nítida a semelhança entre os sexos, não apresentando diferença estatística. (GRÁFICO 1).

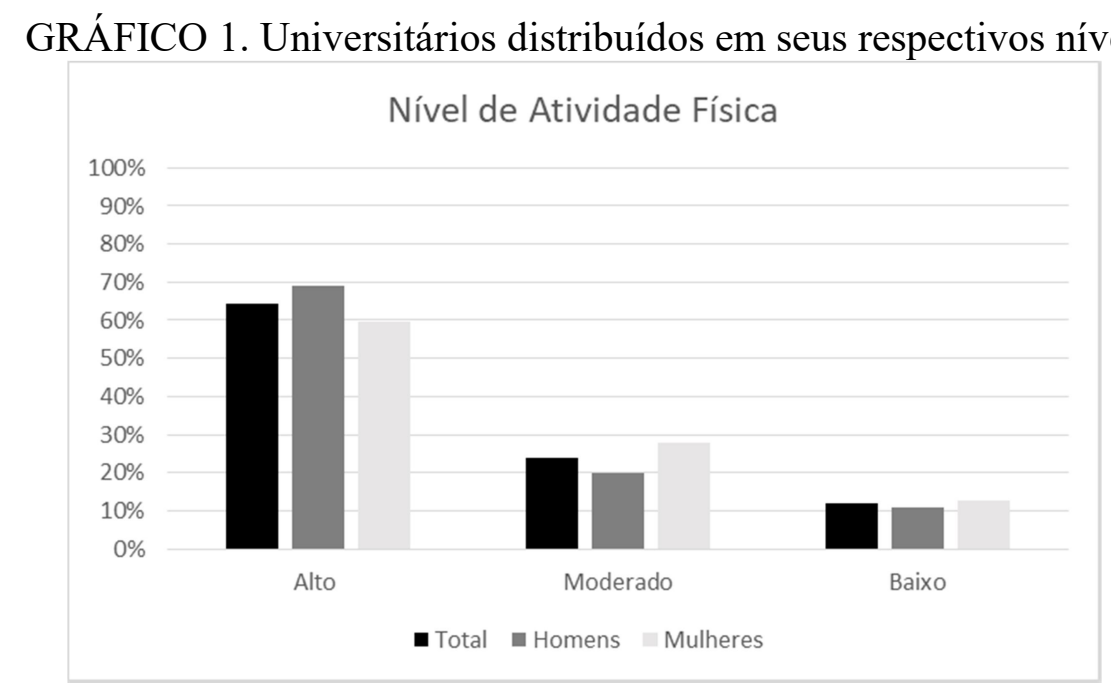

O tempo na posição sentada durante um dia normal da semana apresentou uma média de $6,29 \pm 3,7$ horas. Já o tempo sentado durante um dia do fim de semana se mantém quase o mesmo $6,34 \pm 4,29$ horas.

Quando proposto uma autoavaliação sobre a atual condição de saúde, os participantes em maioria se classificaram entre "Excelente" 28,4\%, "Muito Boa" 33,8\% e "Boa" 30,9\%, ainda, pequena parcela julgou estar "Regular" 4,9\% e "Ruim" $2 \%$.

Quanto ao consumo de álcool, todos universitários relataram consumir em alguma medida, porém, a grande maioria $(91,7 \%)$ apresentou uma classificação "Não Abusiva" do consumo. Apenas 8,3\% estavam classificados como consumo "Abusivo". No entanto, 24,5\%

Persp. online: biol. \& saúde, Campos dos Goytacazes, 34 (10) 58-67, 2020

https://ojs3.perspectivasonline.com.br 
dos universitários expuseram ter aumentado o consumo de bebidas alcoólicas após entrarem no ES.

O uso da nicotina foi minimamente descrito, onde $96,5 \%$ dos universitários relataram não fumar. Dentre os 3,5\% fumantes, 42,85\% apresentaram uma "Alta Dependência", 14,28\% "Moderada" e 42,87\% "Baixa Dependência". Ainda, quando analisados apenas os fumantes, $32 \%$ declararam aumentar o consumo após entrarem no ES.

\section{DISCUSSÃO}

Neste estudo, os bons resultados encontrados podem ser comparados com os de uma pesquisa realizada em Tocantins, onde universitários apresentaram baixos níveis de sedentarismo e de tabagismo, sendo assim, os autores justificam o desfecho como efeitos dos programas nacionais de saúde (RODRIGUES; CHEIK; MAYER, 2008).

Em uma outra pesquisa que avaliou a prevalência de sedentários recém ingressos e comparou com dados sociodemográficos, os autores destacam dois grupos de risco para o sedentarismo, um deles é ser do sexo feminino e o outro é cursar o período noturno (QUADROS et al., 2009). Ainda, mais pesquisas constam baixos níveis de AF nesta população (SOUSA, 2011). Estes dados foram opostos aos resultados encontrados no presente estudo, onde homens e mulheres apresentaram perfis semelhantes, e ainda, mesmo cursando o período noturno, os estudantes apresentaram boas condições em níveis de AF.

Um estudo longitudinal, com acompanhamento de dois anos, realizado em Portugal, concluiu que estudantes expostos a mais tempo à vida acadêmica possuíam maiores níveis de dislipidemia, sobrepeso e tabagismo quando comparados aos recém ingressos na universidade. Ainda, no geral da mesma pesquisa, foi observado uma prevalência de $80 \%$ de sedentários (BRANDÃO; PIMENTEL; CARDOSO, 2011).

De fato, diferenças nos resultados podem ser desde o delineamento do estudo até a população avaliada, sendo esta fortemente influenciada pela dimensão territorial na qual se encontra, uma vez que pesquisas realizadas em cidades de menor porte possuem resultados divergentes daquelas feitas em grandes centros, como exemplo do tabaco, onde cidades menores e menos industrializadas podem apresentar baixos índices de tabagismo (RODRIGUES; CHEIK; MAYER, 2008). Sendo assim, o presente estudo pode ter sofrido influência territorial, onde a amostra representa o perfil de alunos de um Centro Universitário no interior de São Paulo, desta forma, a pequena dimensão territorial somado a campanhas contra o tabaco podem ter influenciado no alto percentil de não fumantes. Porém, um estudo realizado com universitários da Universidade Federal dos Vales do Jequitinhonha e Mucuri (UFVJM) situado na cidade de Diamantina - MG que é considerada uma cidade pequena, mostrou um maior índice de fumantes $(6,9 \%)$, quando comparado com o presente estudo $(3,5 \%)$ o que talvez conteste hipótese de cidades pequenas sofrerem maior influêcnia das campanhas antitabaco (RIBEIRO et al., 2012).

No presente estudo, mínimas diferenças entre os sexos foram encontradas, desta forma, confirmando o espaço ganho pelas mulheres, tanto nos bons níveis de AF, quanto em sua inserção no mercado de trabalho, onde a quantidade de universitários trabalhando e o tempo de trabalho semanal foram praticamente os mesmos entre os sexos, sendo que há poucos anos atrás, diferentes perfis ainda eram observados (QUADROS et al., 2009).

Persp. online: biol. \& saúde, Campos dos Goytacazes, 34 (10) 58-67, 2020

https://ojs3.perspectivasonline.com.br 
Estudos têm mostrado a influência do avançar da idade como responsável por mudanças de hábitos, onde indivíduos com idade superior a 25 anos apresentaram maiores prevalências de sedentarismo e tabagismo (RODRIGUES; CHEIK; MAYER, 2008). Com isso, apesar do presente estudo ter apresentado bons resultados em relação ao nível de AF e a dependência de drogas lícitas, a baixa média de idade apresentada pela amostra (22 anos) ressalta a importância do acompanhamento destes jovens, uma vez que, o respaldo da literatura aponta a exposição acadêmica e o avançar da idade como potenciais estimuladores para queda desses bons níveis (BRANDÃO; PIMENTEL; CARDOSO, 2011; RODRIGUES; CHEIK; MAYER, 2008).

Em relação ao uso/dependência de drogas lícitas, os baixos índices apresentados podem colaborar para um melhor estado de saúde destes jovens, somado ao fato de uma maior proteção contra doenças sexualmente transmissíveis, onde pesquisas apontam que jovens alcoolizados tendem a realizar sexo sem proteção com maior frequência (HUIZINGA; LOEBER; THORNBERRY, 1993).

Atualmente, existem ferramentas mais precisas para avaliar o nível de AF, dependência alcoólica e do tabaco, sendo estas por meio de veículos altamente tecnológicas como uso de sensores de movimento, por uso de parâmetros bioquímicos e indicadores fisiológicos, porém, questionários e ou testes validados pela literatura permitem uma rápida e barata avaliação, viabilizando os estudos populacionais, e também possuindo alta confiabilidade. Isto faz com que instituições ainda em desenvolvimento na área da pesquisa possam crescer e contribuir para a ciência, mesmo ainda sem possuir laboratórios altamente sofisticados.

Como limitações deste estudo, um número maior de participantes poderia ser recrutado, porém, não sendo possível pela quantidade de avaliadores. Outro fator que poderia ser explorado se dá pela divisão dos estudantes de acordo com a área de estudos, ou seja, exatas, humanas e biológicas. Porém, devido à dificuldade em recrutar quantidades pareadas de alunos de cada área, a amostra seguiu sem esta divisão. Ademais, motivos e/ou barreiras sobre prática de AF e o uso de drogas lícitas poderiam ter sido analisados. Para finalizar, o acompanhamento longitudinal possibilitaria traçar com melhor precisão os impactos da exposição acadêmica na vida dos universitários, sendo assim, abre-se espaço para futuras investigações nesta população, principalmente de caráter longitudinal, a fim de corroborar com a literatura e com a saúde pública.

\section{CONCLUSÕES}

Pode-se concluir que a maioria dos estudantes possuem bons níveis de AF, ainda, é altíssima a quantidade de universitários não fumantes e com baixa dependência alcoólica. Sendo assim, com todo respaldo proveniente da literatura, ainda se faz necessário o acompanhamento destes jovens, para que tais hábitos sejam mantidos. Desta forma, é necessário que políticas públicas e institucionais sejam mantidas, ou ainda incrementadas, a fim de possibilitar um contínuo acesso a informações para que tais hábitos sejam praticados ininterruptamente. 


\section{REFERÊNCIAS}

ANDRADE, A. G.; DUARTE, P. C. A. V.; \& OLIVEIRA, L. G. I levantamento nacional sobre o uso de álcool, tabaco e outras drogas entre universitários das 27 capitais brasileiras. Faculdade de Medicina da Universidade de São Paulo - USP e SENAD. Secretaria Nacional de Políticas sobre Drogas, p. 16-282, 2010.

BRANDÃO, M. P.; PIMENTEL, F. L.; \& CARDOSO, M. F. Impact of academic exposure on health status of university students. Revista de saude publica, v. 45, n. 1, p. 49-58, 2011.

CARMO, J. T.; \& PUEYO, A. A. A adaptação ao português do Fagerström test for nicotine dependence (FTND) para avaliar a dependência e tolerância à nicotina em fumantes brasileiros. Revista Brasileira de Medicina, v. 59, n. 1/2, p. 73-80, 2002.

CONSTANTINO, P.; \& BATISTA, L. DE S. S. Perfil dos usuários de Substâncias psicoativas do CAPS Ad Em 2000 E 2009, Campos Dos Goytacazes, RJ. Biológicas \& Saúde, v. 2, n. 7, 27 out. 2012.

CRISCI, A. R.; OTERO, C. Q.; FAUSTO DE OLIVEIRA, L. F.; VIANNA, N. P.; \& CASTRO, M. P. Poluição tabagística ambiental (pta) e suas consequências no aparelho respiratório e ganho de peso em ratos wistar. Biológicas \& Saúde, v. 5, n. 16, 3 jun. 2015.

DEHEINZELIN, D.; LOURENÇO, M.T.C.; COSTA, C.L.D.; \& YOUNES, R.N. The level of nicotine dependence is an independent risk factor for cancer: a case control study. Clinics, v. 60, n. 3, p. 221-226, 2005.

EWING, J. A.; \& ROUSE, B. A. Identifying the hidden alcoholic. In: Program and abstracts of the 29th Internacional Congress on Alcohol and Drug Dependence. Sidney, Australia.1970.

FONTES, A.C.D.; \& VIANNA, R.P.T. Prevalência e fatores associados ao baixo nível de atividade física entre estudantes universitários de uma universidade pública da região Nordeste - Brasil. Revista Brasileira de Epidemiologia, v. 12, n. 1, p. 20-29, 2009.

HUIZINGA, D.; LOEBER, R.; \& THORNBERRY, T. Longitudinal study of delinquency, drug use, sexual activity, and pregnancy among children and youth in three cities. Journal of Quantitative Criminology, v. 49, n. 4, p. 90-96, 1993.

MALBERGIER, A.; \& OLIVEIRA JUNIOR, H. P. Dependência de tabaco e comorbidade psiquiátrica. Archives of Clinical Psychiatry (São Paulo), v. 32, n. 5, p. 276-282, 2005.

MASUR, J.; \& MONTEIRO, M. G. Validation of the "Cage" alcoholism screening test in a brazilian psychiatric inpatient hospital setting. Brazilian Journal of Medical and Biological Research, v. 16, n. 3, p. 215-218, 1983.

MATSUDO, S.; ARAÚJO, T.; MATSUDO, V.; ANDRADE, D.; ANDRADE, E.; OLIVEIRA, L. C., \& BRAGGION, G. International physical activity questionnarie (IPAQ): study of validity and reliability in Brazil. Revista Brasileira de Atividade Física e Saúde, v. 6, n. 2, p. 6-18, 2001. 
MILLAR, W. J.; \& LOCKER, D. Smoking and oral health status. Journal of the Canadian Dental Association, v. 73, n. 2, 2007.

ODO, S. A.; ARAUJO, A.C.; SANTOS, A.F.D.; TOLEDO, F.C.; YONAMINE, M.; SILVA, O.A.; \& LEITE, M.D.C. Indicações e limites das análises toxicológicas para substâncias psicoativas. Revista de Psiquiatria Clínica, v. 27, n. 1, p. 50-56, 2000.

OLIVEIRA; A.F.; VALENTE, J.G.; \& LEITE, I.C. Aspectos da mortalidade atribuível ao tabaco : revisão sistemática Aspects of tobacco attributable mortality: systematic review. Vide Science Technique Et Applications, v. 42, n. 2, p. 335-345, 2008.

OMS. Prevenção de doenças crônicas um investimento vital. OMS, p. 6-11, 2005. Disponível em: https://www.who.int/chp/chronic_disease_report/contents/en/. Acesso em: 10 janeiro 2020 .

PITANGA, F. J. G.; \& LESSA, I. Prevalência e fatores associados ao sedentarismo no lazer em adultos. Cadernos de Saúde Pública, v. 21, n. 3, p. 870-877, 2005.

QUADROS, T. M. B.; PETROSKI, E.L.; SILVA, D.A.S.; \& GORDIA, A.P. Prevalência de sedentarismo em universitários brasileiros: associação com variáveis sociodemográficas. Revista de Salud Publica (Bogota), v. 11, n. 5, p. 724-733, 2009.

REITSMA, M. B. FULLMAN, N.; NG, M.; SALAMA, J. S.; ABAJOBIR, A.; ABATE, K.H.; ... \& ADEBIYI, A. O. Smoking prevalence and attributable disease burden in 195 countries and territories, 1990-2015: a systematic analysis from the Global Burden of Disease Study 2015. The Lancet (London, England), v. 389, n. 10082, p. 1885-1906, 2017.

RIBEIRO, V.B.; DE MELO IRENO, M.S.; MARTINS, F.L.M.; \& DE LIMA, V.P. Prevalência de jovens universitários tabagistas e suas inter-relações. ConScientiae Saúde, v. 11, n. 1, p. 9-16, 2012.

RODRIGUES, E.S.R.; CHEIK, N.C.; \& MAYER, A.F. Nível de atividade física e tabagismo em universitários. Revista de Saúde Pública, v. 42, n. 4, p. 672-678, 2008.

SILVA, L.R.V.E.; MALBERGIER, A.: STEMPLIUK, V.D.A.; \& ANDRADE, A.G.D. Fatores associados ao consumo de álcool e drogas entre estudantes universitários. Revista de Saúde Pública, v. 40, n. 2, p. 280-288, 2006.

SOUSA, T. F. Inatividade física em universitários brasileiros : uma revisão sistemática. Revista Brasileira de Ciências da Saúde, p. 47-55, 2011.

TROLLE-LAGERROS, Y.; MUCCI, L.A.; KUMLE, M.; BRAATEN, T.; WEIDERPASS, E.; HSIEH, C.C.; ... \& ADAMI, H.O. (Physical activity as a determinant of mortality in women. Epidemiology, v. 16, n. 6, p. 780-785, 2005. 